\title{
Magnetic Resonance Imaging of a Benign Phyllodes Tumor of the Breast
}

\author{
Ravikanth Balaji Krishnankutty N. Ramachandran \\ Regional Cancer Centre, Imageology Division, Medical College PO, Trivandrum, India
}

\section{Key Words}

Phyllodes tumor - Magnetic resonance imaging . Magnetic resonance spectroscopy · Slit-like spaces

\section{Summary}

Background: The aim of this study was to evaluate a phyllodes tumor of the breast with magnetic resonance imaging (MRI) and magnetic resonance spectroscopy. Methods: MRI of the breasts was performed using a phased array double breast coil with spectroscopic capability on a 1.5T MR scanner. Post-contrast sequential imaging was done, and the subtracted images were evaluated. Timesignal intensity curves were obtained. MR spectroscopy using BREASE software was also performed. Results: MRI combined with MR spectroscopy was used in the diagnosis and characterization of a phyllodes tumor of the breast. Conclusion: MRI and MR spectroscopy may offer an in vivo imaging technique for the characterization of phyllodes tumors of the breast.

\section{Introduction}

Phyllodes tumors are rare breast neoplasms representing only $0.3-1 \%$ of all breast tumors [1]. These tumors resemble fibroadenomas so closely that their radiological and histopathology features often overlap, more so in cases of giant fibroadenoma [2]. Phyllodes tumors grow rapidly to large sizes with a median size of $4-5 \mathrm{~cm}$ while most fibroadenomas rarely exceed $3 \mathrm{~cm}$ in size [3]. Magnetic resonance imaging (MRI) coupled with dynamic gadolinium contrast study plays an im-

\author{
Schlüsselwörter \\ Phylloidtumor · Magnetresonanztomographie · \\ Magnetresonanzspektroskopie · Schlitzartige \\ Zwischenräume
}

\section{Zusammenfassung}

Hintergrund: Ziel dieser Studie war die Untersuchung eines Phylloidtumors der Brust mithilfe der Magnetresonanztomographie (MRT) und Magnetresonanzspektroskopie. Methoden: Das Brust-MRT wurde mit einer spektroskopisch einsetzbaren, "phased array" MammaDoppelspule an einem 1.5T-MRT- Scanner durchgeführt. Die Messungen erfolgten in Form von sequentiellen Postkontrast-Aufnahmen, und die subtrahierten Aufnahmen wurden bewertet. Signal-Zeit-Kurven wurden erstellt. Die magnetresonanzspektroskopische Analyse wurde mit der BREASE-Software durchgeführt. Ergebnisse: MRT und Magnetresonanzspektroskopie wurden eingesetzt, um einen Phylloidtumor der Brust zu diagnostizieren und zu charakterisieren. Schlussfolgerung: MRT und Magnetresonanzspektroskopie könnten gemeinsam eine bildgebende In-vivo-Untersuchungsmethode zur Charakterisierung von Phylloidtumoren der Brust bilden.

portant role in the diagnosis as well as the distinction of benign phyllodes tumors from borderline and malignant types. We describe MRI features of a benign phyllodes tumor of the breast in a 25-year-old woman.

\section{Case Report}

A 25-year-old woman presented with a progressively enlarging painless mass in the left breast. Clinical examination revealed a large, firm, non-

\section{KARGER}

Fax +497614520714

Information@Karger.de

www.karger.com (c) 2009 S. Karger GmbH, Freiburg 
Fig. 1. A Axial T1WI shows a hypointense mass with tiny hyperintense foci of hemorrhage. B On axial T2WI, the mass shows high signal intensity with hyperintense slit-like fluid-filled spaces. C Axial STIR sequence showing hyperintense mass with increased signal spaces within.

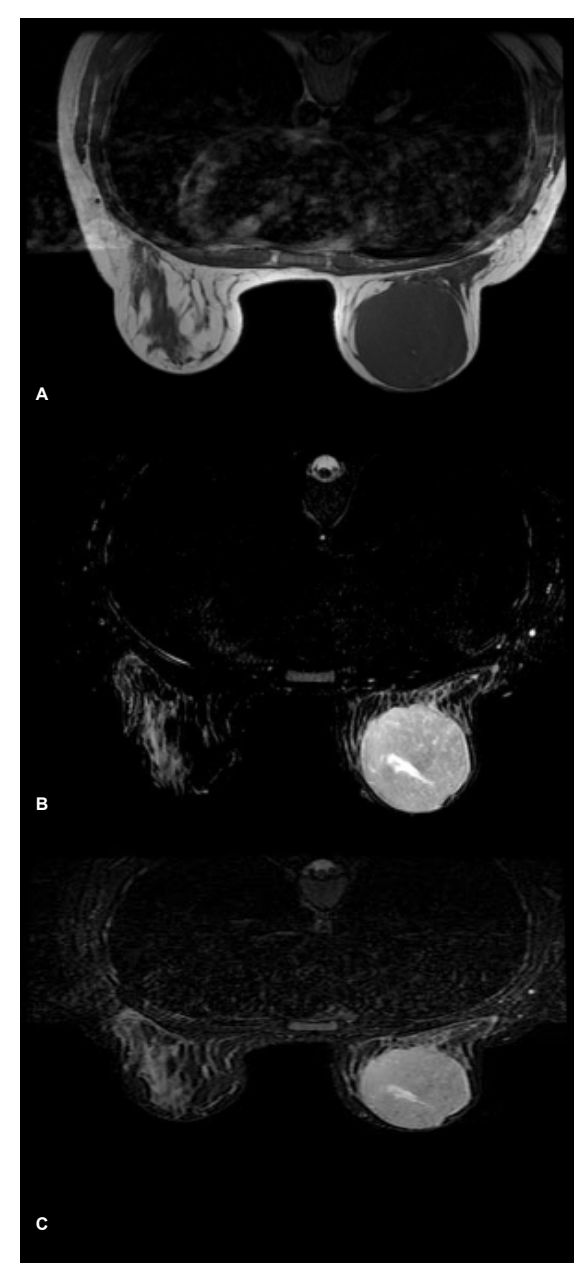

tender, slightly mobile mass almost involving the entire breast. The skin over the swelling was stretched and taut with no nipple retraction. Axillary nodes were not enlarged. Routine mammograms showed a large well defined mass involving the left breast. There were no benign or suspicious malignant microcalcifications or architectural distortion. The right breast was unremarkable. No enlarged axillary nodes were noticed. Sonography revealed a well-marginated uniformly hypoechoic mass with minimal posterior acoustic enhancement.

MRI was performed on a 1.5T HDx Twin Speed scanner (GE Medical Systems, Milwaukee, WI, USA) using a dedicated phased array double breast coil with spectroscopic capability. MRI revealed a large well-circumscribed predominantly hypointense mass on T1WI (fig. $1 \mathrm{~A}$ ) measuring $6.2 \times 6 \mathrm{~cm}$, almost involving the entire left breast. Tiny hyperintense foci were seen in the mass representing hemorrhage. The lesion showed increased signal intensity on T2WI (fig. 1 B) and appeared hyperintense on STIR (short tau inversion recovery) sequence (fig. 1 C). Slit-like hyperintense foci were noted in the center of the lesion on T2WI FS (fatsuppressed). A large leash of vessels was seen anteriorly leading into the axillary vein. There was no infiltration into the chest wall or subcutaneous tissues. On diffusion-weighted imaging (DWI), the mass displayed restricted diffusion with increased apparent diffusion coefficient (ADC) values. On multiphase post-gadolinium augmented study, intense enhancement was seen with no washout in late phases (fig. 2 A). Time-signal intensity kinetic curves reflected the fast initial contrast enhancement with no washout in the later phases (Type Ib type of enhancement curve) (fig. 2 B). 1H MR spectroscopy performed using BREASE software (GE Medical Systems) revealed a very weak choline signal at $3.2 \mathrm{ppm}$ which indicated the benign nature of the neoplasm (fig. 3).

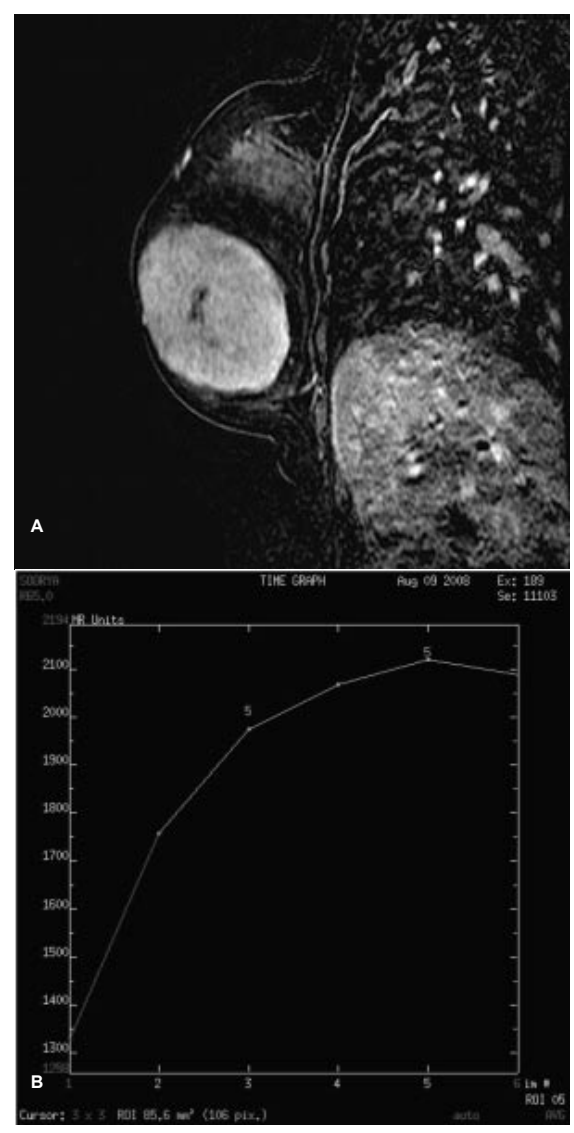

Fig. 2. A Sagittal post-gadolinium subtraction image shows intense enhancement B Time-signal intensity kinetic curve showing Type Ib enhancement - progressive enhancement with initial rapid enhancement.

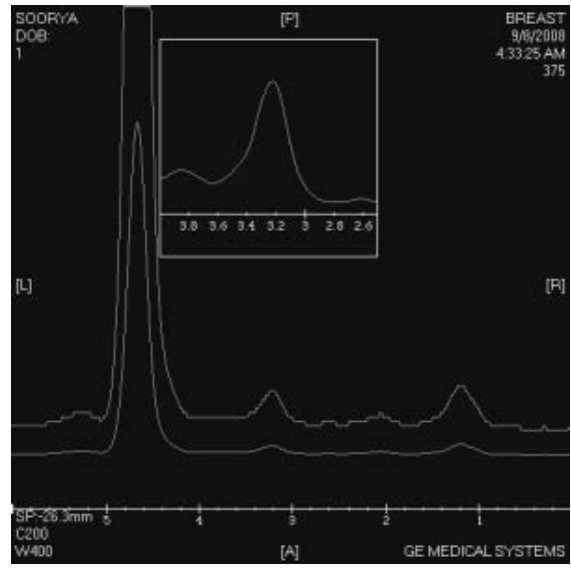

Fig. 3. $1 \mathrm{H}$ spectroscopy showing insignificant choline resonance at $3.2 \mathrm{ppm}$.

Simple excision of the tumor was carried out. The mass was well encapsulated, and the cut surface appeared grayish white. Cleft-like spaces corresponding to the hyperintense foci on T2WI were seen, some showing hemorrhagic content. Histopathological analysis showed highly cellular stroma with hyperplastic epithelial content.

\section{Discussion}

Phyllodes tumors are rare neoplasms of the breast that arise from the periductal stroma. Three categories of phyllodes tumor have been described based on neoplastic potential: be- 
nign, intermediate (borderline), and malignant. Because of their rapid growth, these tumors do not show any calcification [2]. Histopathologically, papillary projections of epithelium-lined stroma are seen, which appear as leaf-like masses; hence the term 'phyllodes' which is Greek for leaf-like. Farria et. al. [4] and Kinoshita et al. [5] described MRI features of benign phyllodes tumors as masses with well-circumscribed margins, internal septations, high signal intensity on fat-suppressed T2-weighted sequences, and rapid initial enhancement on dynamic imaging. In our case, MRI showed a lobular mass with smooth margins appearing hypointense on T1WI and having high signal intensity on T2WI. Hyperintense foci on T1WI may represent hemorrhage. On T2WI FS and STIR sequences, hyperintense fluid-filled slit-like spaces were seen in the central part of the mass, which favored a diagnosis of phyllodes tumor. Time-signal intensity kinetic curves revealed a rapid initial intense enhancement pattern with no wash-out on contrast-enhanced dynamic imaging. All MRI features observed in our case were consistent with the imaging characteristics of a benign phyllodes tumor [4-9]. The fast initial intense enhancement pattern without wash-out is similar to the dynamic imaging pattern reported by Ogawa et al. [8] for benign phyllodes tumor. The rapid dynamic enhancement pattern may be due to increased angiogenesis in phyllodes tumors [3]. The cells are believed to produce angiogenesis factors which increase cellularity of the mesenchymal and epithelial components of the tumor [7]. Inhomogeneous signal intensities on T1WI and T2WI relating to the presence of hemorrhage and cystic areas with internal septations have been reported in 3 cases of phyllodes tumor [10]. Cystic spaces present within these tumors may offer a clue for the differentiation of benign from borderline and malignant phyllodes tumors [7]. Cystic spaces with smooth margins and exhibiting homogeneous hyperintense signal on T2WI are consistent with benign tumors while irregular margins and heterogeneous signal intensities would favor a borderline or malignant lesion. Heterogeneous inner structure was more often seen in malignant phyllodes tumors, probably due to their rapid growth resulting in irregular structure [7]. Dynamic MRI pattern is helpful in differentiating benign from malignant lesions $[6,7,11]$.

Another important tool in the radiologist's repertoire to identify a malignant process is proton $(1 \mathrm{H}) \mathrm{MR}$ spectroscopy. Elevation of choline peak (resonance at $3.2 \mathrm{ppm}$ ) resulting from increased membrane turnover by rapidly proliferating tumor cells has been considered an indicator of malignancy. The spectrum obtained in our case revealed a weak choline signal at $3.2 \mathrm{ppm}$ which was insignificant, confirming the benignity of the mass.

In conclusion, a large mass with well-defined margins appearing heterogeneous on T2WI and showing hyperintense fluid-filled slit-like spaces on T2WI FS and STIR sequences, and rapid initial contrast enhancement on dynamic imaging with no washout, is suggestive of benign phyllodes tumor. Surgical excision is the definitive treatment when the tumor size is large. The chance of local recurrence is high with a reported incidence of $20 \%$.

\section{Conflict of Interest}

The authors have no conflict of interest to declare.

\section{References}

1 Rosewell MD, Perry RR, Hsju JG, Barranco SC: Pyllodes tumors. Am J Surg 1983;165:376-379.

$\checkmark 2$ Page JE, Williams JE: The radiolgic features of phyllodes tumor of the breast with clinico-pathological correlation. Clin Radiol 1991;44:8-12.

3 Cohn-Cedermark G, Rutqvist LE, Rosendahl I, Silfversward C: Prognostic factors in cystosarcoma phyllodes: a clinicopathological study of 77 patients. Cancer 1991;68:2017-2022.

4 Farria DM, Gorczyea DP, Barsky SH, Sinha S, Basset LW: Benign phyllodes tumor of the breast; MR imaging features. AJR 1996;167:187-189.

5 Kinoshita T, Fukutomi T, Kubochi K: Magnetic resonance imaging of benign phyllodes tumor of the breast. Breast J 2004;10:232-236.
6 6 Wurdinger S, Herzog AB, Fischer DR, Marx C, Raabe G, Schneider A, Kaiser WA: Differentiation of phyllodes breast tumors from fibroadenomas on MRI. AJR 2005;185:1317-1321.

7 Yabuchi H, Soeda H, Matsuo Y, Okafuji T, Eguchi T, Sakai S, Kuroki S, Tokunaga E, Ohno S, Nishiyama K, hatakenaka M, Honda H: Phyllodes tumor of the breast: correlation between MR findings and histologic grade. Radiology 2006;241:702-709.

8 Ogawa Y, Nishioka A, Tsuboi N, Yoshida D, Inomata T, Yoshida S, Moriki T, Toki T: MR appearances of benign phyllodes tumor of the breast in a 20-year-old woman. Radiat Med 1997;15:247-250.
9 Heywang-Kobrunner SH: Contrast-enhanced magnetic resonance of the breast. Invest Radiol 1994;29: 94-104.

10 Francescheni G, D’Ugo D, Masseti R, Palumbo F, D'Alba PF, Mule A, Costantini M, Belli P, Picciocchi A: Surgical treatment and MRI in phyllodes tumors of the breast: our experience and review of the literature. Ann Ital Chir 2005;76:127-140.

11 Kuhl CK, Mielcareck P, Klaschik S, et al.: Dynamic breast MR imaging: are signal intensity time course data useful for differential diagnosis of enhancing lesions? Radiology 1999;211:101-110. 